\title{
Le roseau protecteur
}

Techniques et symboliques d'une plante dans le Sud marocain (Tafilalt)

Marie-Luce Gélard

\section{(2) OpenEdition}

12 Journals

Édition électronique

URL : https://journals.openedition.org/tc/2742

DOI : $10.4000 /$ tc. 2742

ISSN : 1952-420X

Éditeur

Éditions de l'EHESS

Édition imprimée

Date de publication : 12 décembre 2007

Pagination : 61-84

ISSN : 0248-6016

\section{Référence électronique}

Marie-Luce Gélard, «Le roseau protecteur », Techniques \& Culture [En ligne], 48-49 | 2007, mis en ligne le 20 juin 2010, consulté le 29 septembre 2022. URL : http://journals.openedition.org/tc/2742 ; DOI : https://doi.org/10.4000/tc. 2742 


\section{Marie-Luce Gélard*}

\section{Le roseau protecteur Techniques et symboliques d'une plante dans le Sud marocain (Tafilalt)}

Ce texte propose l'examen des différents emplois du roseau (Arundo donax) et illustre les représentations qui lui sont associées dans la région saharienne de Merzouga (Sud-Est marocain). Ainsi, se croisent et se répondent à la fois des données techniques, dans l'habitat, l'agriculture, l'élevage du bétail, etc. et des données symboliques (utilisations rituelles, représentations, manifestations de la fécondité) où la plante est pensée et perçue comme le vecteur de fonctions rituelles toutes dérivées de la protection. Le roseau protège de la chaleur et de l'ensablement les denrées nourricières du groupe (cultures et reproduction du cheptel animal), permettant leur fertilisation via les rites de rogations pour la pluie où il est un élément central. Le roseau est aussi un "instrument" de survie du nourrisson par la mesure des modifications de son corps (croissance) et enfin, c'est encore lui qui permet d'éloigner certains maux de la communauté par son pouvoir d'attraction (rituel sacrificiel notamment).

Roseau, Aït Khebbach, Sahara, corps, représentations.

Dans le Tafilalt, oasis située entre les oueds Ghéris et Ziz¹, le roseau ${ }^{2}$ occupe une place privilégiée, d’un point de vue technique - son utilisation est d'une

\footnotetext{
* Anthropologue, Maître de conférences, Université Paris Descartes, CNRS, UMR 8099, Langues-musiquessociétés.

Je remercie très chaleureusement Geneviève Bédoucha, Tatiana Benfoughal et Sébastien Boulay pour leur relecture de ce texte.

${ }^{1}$ Le Tafilalt désigne la région du Sud-Est marocain englobant tout à la fois l'oasis, l'erg Chebbi (formation dunaire qui précède le grand erg occidental) et la vallée du Ziz jusqu'au Haut Atlas.

2 Arundo donax L., il s'agit d'une graminée dite «à caractère primitif ", que les botaniques classent dans le groupe des phragmitiformes. «Plante vivace à rhizome rampant, très ramifié, émettant des tiges nombreuses, élevées (de $60 \mathrm{~cm}$ à deux mètres), dures et luisantes ; feuilles glauques, à ligule courte et ciliée [...]. Espèce cosmopolite, surtout représentée au Sahara par une forme à feuilles courtes, raides et piquantes, un peu
} 
grande diversité-, mais aussi et surtout, d'un point de vue symbolique. Son emploi domestique est multiple : terrasses des habitations, enclos du bétail, éléments amovibles des métiers à tisser, etc. Il est aussi utilisé dans différentes pratiques rituelles: procédure symbolique pour préserver la virginité des jeunes filles, coupe du cordon ombilical, pratiques sacrificielles. Ses qualités de souplesse et de grande résistance font du roseau un élément essentiel qui ne semble pas avoir trouvé d’autre élément de substitution tel le plastique qui supplante désormais une partie des productions matérielles sahariennes traditionnelles : poteries, vanneries (Benfoughal 1996).

Écartant le projet de dresser le tableau exhaustif des différents usages du roseau dans le Tafilalt, cette contribution entend illustrer les liens entre certaines des propriétés techniques de la plante et les représentations symboliques qu'elle sous-tend, doublement signifiantes dans différents rituels.

\section{De l'utilité du roseau ou l'insubstituable matière : babitat, agriculture et élevage en milieu sabarien}

Dans les oasis présahariennes du Sud marocain, caractérisées par un habitat ksourien et "post-ksourien ${ }^{3}$ ", les terrasses des maisons sont construites à laide de roseaux. Dans de rares exceptions, le toit est parfois réalisé en palmes, c'est le cas des habitations les plus anciennes (fig. I). Si deux ou trois personnes suffisent à l'édification des maisons ${ }^{4}$ selon la traditionnelle technique du coffrage ${ }^{5}$, les terrasses, elles, requièrent un travail collectif et imposent pour des raisons techniques, la présence d'une dizaine de personnes. En effet, lorsque les murs sont achevés et que les madriers sont

enroulées en long, à tiges plus courtes que dans le roseau habituel d'Europe. Lits des torrents, gueltas, un peu partout au Sahara septentrional, occidental et central » (Ozenda 1983 : 176).

3 Cette terminologie est usitée par la plupart des géographes pour définir les différentes agglomérations qui ont remplacé l'habitat traditionnel du ksar, village fortifié.

${ }^{4}$ Les villages des Aït Khebbach sont assez récents (milieu du XXe siècle) ce qui explique l'absence d'élévation en hauteur des maisons, caractéristique de l'habitat ksourien et des nécessités des fonctions défensives. Pour plus de détails sur l'origine et les conditions historiques du ksar en milieu oasien marocain voir J. Hensens (1969 : 83-107).

5 Les demeures sont construites à l'aide de coffrages de bois remplis de terre. Les murs sont ensuite recouverts de pisé (intérieur et extérieur). Les constructions individuelles en béton sont encore l'exception au sein des villages même si elles commencent à apparaitre (deux habitations de ce type à Merzouga). À cela plusieurs raisons, d'une part les coûts des matériaux et de leur transport (la terre utilisée étant présente sur place, celle des coffrages et l'argile du pisé), et d'autre part, la nature peu isolante de ces matières. 
posés, on procède à la confection du plafond par un assemblage de roseaux, liés les uns aux autres à l'aide de liens serrés. Entre les poutres ${ }^{6}$ est placé, parallèlement, un roseau robuste de gros diamètre ${ }^{7}$ auquel on attache des tiges de roseaux ordinaires disposées perpendiculairement. Chacune de ces tiges étant liée séparément. Dans la mesure où il convient de serrer fermement l'assemblage, une personne ne peut s'occuper simultanément de plus de trois roseaux, raison pour laquelle et en fonction de la taille de la pièce, cette opération nécessite la présence conjointe d'une dizaine de personnes. Ce travail est très souvent réalisé par des adolescents (filles ou garçons) sous la responsabilité d'un ou deux adultes. Une fois le plafond de roseau achevé ${ }^{8}$, ce dernier est recouvert d'une bâche de plastique puis de terre argileuse. La terrasse servira l'été de dortoir collectif à l'ensemble des membres de la famille, ainsi qu’au séchage de différentes denrées (dattes, par exemple). On utilise surtout le roseau car il assure une isolation thermique efficace ${ }^{9}$ pour lutter contre des températures dépassant souvent $50^{\circ} \mathrm{C}$ durant les mois de juin et de juillet.

Avant la réalisation de la toiture, les roseaux doivent être débarrassés de leurs feuilles à l'aide d’une faucille. En effet, il est nécessaire de retirer du roseau, le chaume soit la feuille, la gaine de la feuille et la ligule. Pour des raisons esthétiques, dans la mesure où de lintérieur des pièces, le plancher de roseaux est visible, il convient de ne laisser apparentes que les parties lisses des tiges ${ }^{10}$.

\footnotetext{
${ }^{6}$ Il s'agit de rondins de bois d'eucalyptus importés du Nord, d'un diamètre d'une quinzaine de centimètres.

${ }^{7}$ Les roseaux les plus grands proviennent de la région de Meknès, ceux plus ordinaires sont produits dans les palmeraies du village d'Aoufouss à une centaine de kilomètres au nord.

8 Dans l'Aurès (Algérie), les terrasses sont réalisées de manière identique, le roseau étant remplacé par des branches de lauriers rose placées sur les solives (Gaudry 1929 : 21).

${ }^{9}$ Le roseau est aujourd'hui testé pour certaines applications de nouvelle technologie, notamment dans la filière du bâtiment pour la réalisation de panneaux d'isolation.

10 Dans certaines maisons mais surtout dans les auberges touristiques des alentours, les plafonds sont aussi constitués en roseau mais en modifiant le placement de ces derniers afin de réaliser des damiers parfois très complexes voire colorés (fig. 2). Cette tâche est très longue et nécessite un important travail de découpe préalable puisque certains éléments de roseau ne mesurent qu'une vingtaine de centimètres.
} 


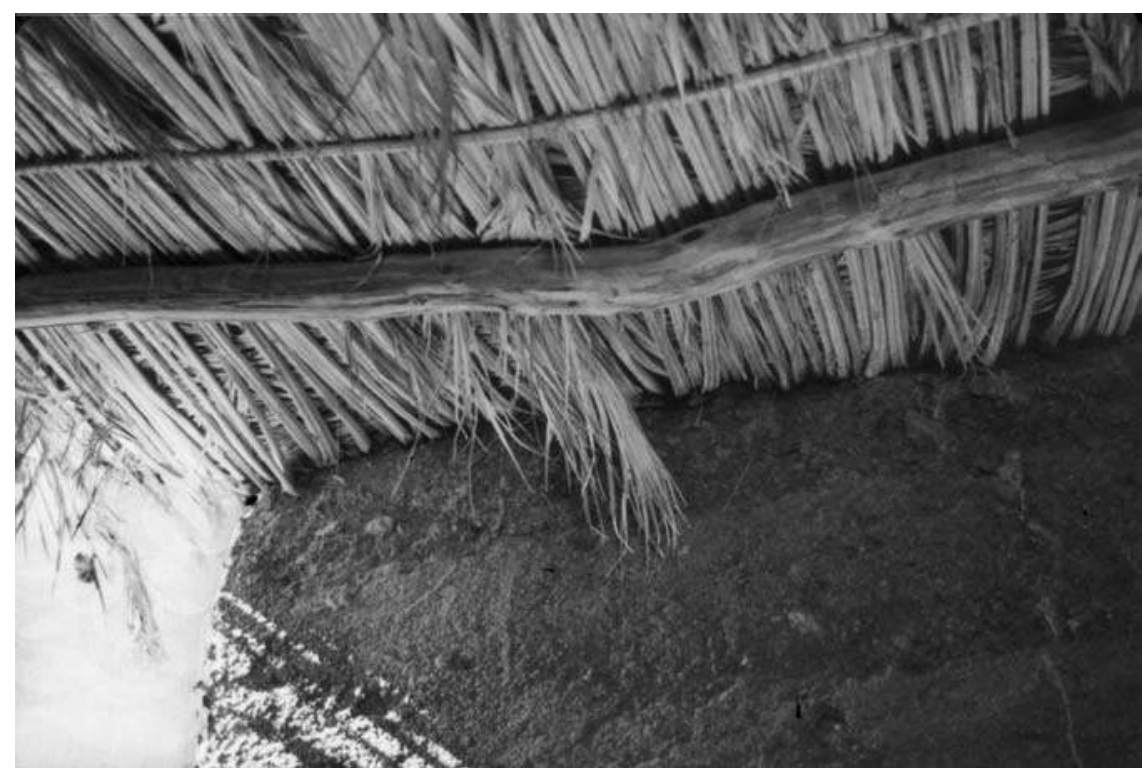

Figure 1. Toiture en palmes dans une demeure ruiniforme de Taqucht. Construction datant des années 1960 (cliché M.-L. Gélard, 2005).

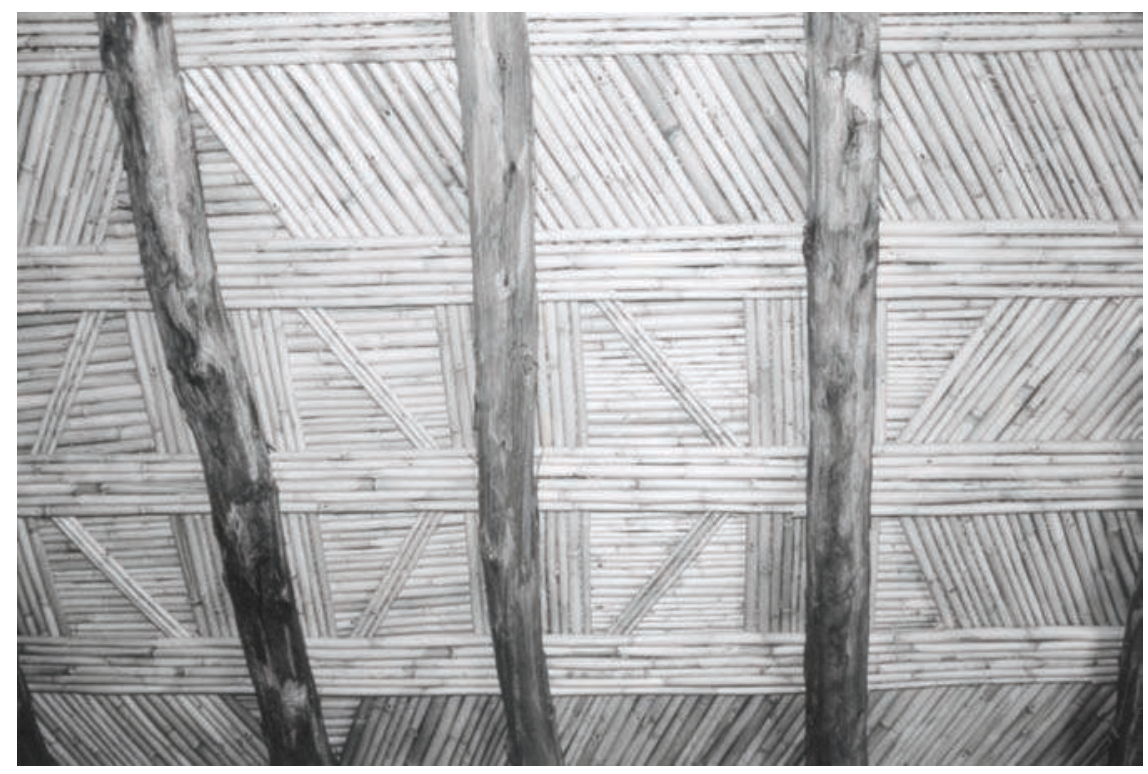

Figure 2. Toiture en roseaux vue de l'intérieur, village d'Hassy Lbyed (cl. M.-L. Gélard, 2005). 
Cette activité de préparation des cannes de roseau est très souvent prise en charge par les jeunes, parfois par les femmes. Dans ce cas, celles-ci se réunissent entre voisines, c'est l'occasion d'entamer une activité collective, moment d'échange et de socialisation intense (plaisanteries, chants, joutes diverses) qui exclut les hommes. Les feuilles des roseaux servent ensuite de fourrage pour le bétail.

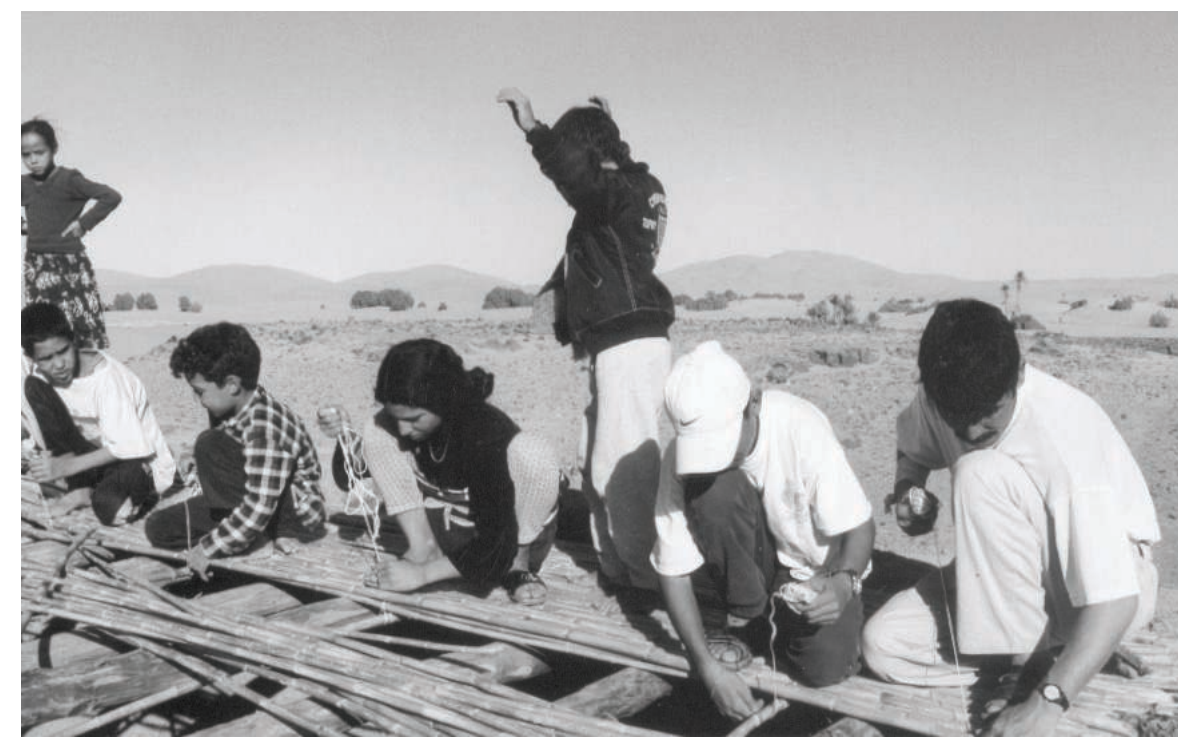

Figure 3. Construction d'une terrasse. Village de Taqucht (cliché M.-L. Gélard, 2003).

Très généralement, les différentes terrasses d'une nouvelle construction sont réalisées à quelques jours d'intervalle, les roseaux à préparer sont alors très nombreux ce qui nécessite un véritable travail collectif. Les paquets de roseaux d'une soixantaine de cannes environ sont déposés à lintérieur des habitations en construction et dressés vers le ciel. Tout le monde est ainsi informé de la fabrication à venir des terrasses. Cette lisibilité est très importante, c'est elle qui permet aux jeunes de se manifester soit pour préparer les roseaux, soit pour participer à la construction finale (fig. 3 ). 
Ces activités collectives sont fréquentes en milieu rural berbérophone ${ }^{11}$. À Merzouga, elles concernent différents domaines : agriculture (récolte et vannage), système d'irrigation (nettoyage des conduits et construction d'adductions), tissage, etc. On assiste alors à une stricte séparation des catégories de sexe. Or, dans le cas de l'édification des planchers en roseaux, dans la mesure où ce sont des adolescents qui les réalisent, la distinction des genres n'apparaît pas. C'est d'ailleurs l'occasion pour les jeunes hommes et les jeunes filles (jusquà une douzaine d'années environ), de sobserver mutuellement, les adultes présents attisant, de leurs plaisanteries, la gêne des jeunes filles et des jeunes garçons.

Compte tenu de la généralisation de l'emploi des roseaux pour les constructions, c'est à cet effet quill est le plus usité. Son deuxième emploi concerne l'agriculture vivrière.

Le roseau, une barrière protectrice contre le sable

Roseau et palmes sont utilisés pour éviter la progression dunaire en direction des terres cultivées (fig. 4).

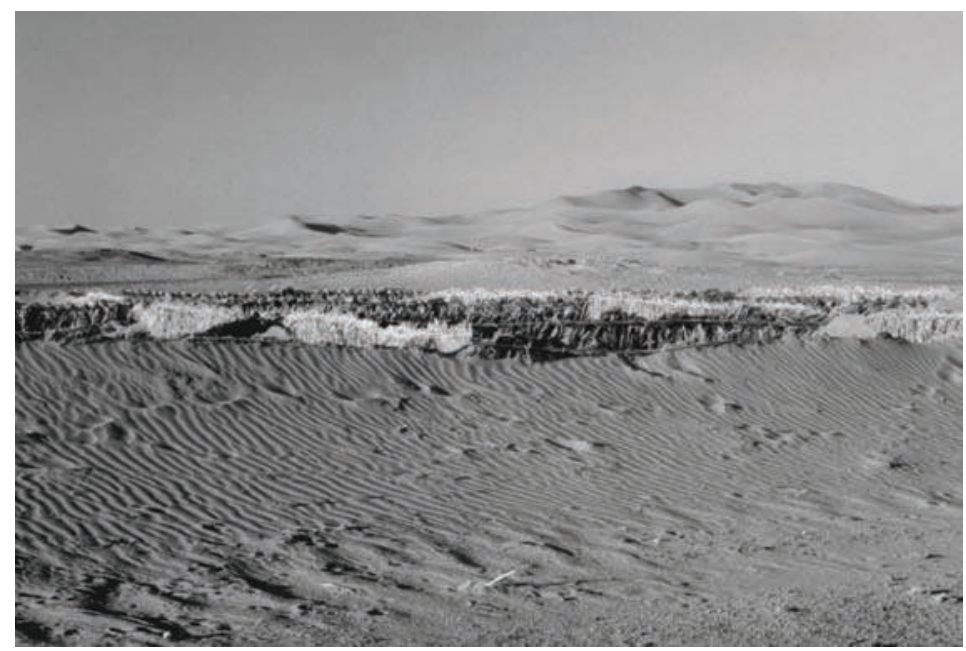

Figure 4. Barrière de protection de la palmeraie, Merzouga (cl. M.-L. Gélard 2004)

11 À propos des Maures de Mauritanie, voir l'analyse par Sébastien Boulay (2003) des inversions des valeurs masculines et féminines à l'occasion de ces travaux collectifs. 
À Merzouga, il existe une palmeraie principale ${ }^{12}$ et plusieurs palmeraies secondaires de plus petites dimensions dont certaines sont aujourdhui abandonnées en raison de lavancée du sable ${ }^{13}$. Pour protéger les jardins (igighen) de l'ensevelissement, on dispose tout autour des zones cultivées et irriguées des barrières de roseaux et de palmes qui retiennent le sable. Cependant, en raison du manque d'eau, on ne plante pas le roseau dont les racines empêcheraient aussi l'érosion du sol, il n'est utilisé que coupé et fiché dans le sable. Il faut refaire régulièrement la barrière derrière laquelle laccumulation dunaire se produit. Les palmes proviennent des palmiers des différentes oasis, mais ne sont pas suffisantes. En effet, ces dernières sont aussi et surtout employées comme combustible dans les différents fours à pain collectifs du village. Les nécessités techniques de la cuisson du pain, associant un combustible lent à un plus rapide au moment de la levée, rendent indispensable l'utilisation quotidienne de ces palmes. L'absence du roseau comme combustible est notoire. Dans plusieurs des fractions de la tribu des Ait Khebbach, brûler un roseau est considéré comme un acte grave. C'est le cas essentiellement des Ait Bourk, divisés en Aït Adiya et Aït Ali. Un roseau ayant poussé sur la tombe de l'ancêtre éponyme de la fraction, Sidi Ali Ait Bourk, brûler un roseau porte malheur. On rapporte de nombreuses anecdotes à ce propos. Ainsi, récemment, une jeune fille des Aït Bourk utilisa un morceau de roseau pour cuire son pain au four. Lorsqu'elle retira le pain du four, ce dernier sortit entièrement noir (ifughd iya unyel) ${ }^{14}$. Lorsque les familles des Aït Bourk achèvent des travaux de construction, le reste des roseaux non utilisés est déposé à l'extérieur des habitations, loin des réserves de bois (fig. 5) afin de ne jamais servir de combustible.

Dans les zones sub-sahariennes, Edmond Bernus (1985: 207) relève l'emploi du terme afarag pour désigner la clôture des champs irrigués, par

12 Comme pour l'ensemble des tribus Ait Atta (ensemble confédéré auquel se rattache la tribu des Ait Khebbach), sociétés agro-pastorales, la sédentarisation dans un lieu donné s'effectue par une occupation collective d'un lieu, l'acte fondateur étant généralement constitué par la création d'une adduction d'eau (à Merzouga il s'agit de foggaras qui s'étendent sur une dizaine de kilomètres, l'eau provenant de l'erg) permettant de créer une palmeraie. Le village est ensuite construit à proximité, plus loin les terres collectives bénéficiant d'une irrigation par le débordement des eaux de pluie (oued). En d'autres termes, on retrouve la distribution spatiale tripartite caractéristique du statut foncier des Ait Atta décrite par David Hart (1981).

13 Cultivé dans tout le Sud marocain depuis fort longtemps, le palmier dattier n'est cependant pas un constituant de la végétation climatique du pays. Voir notamment M. Mohammed Fennane (1987 : 171-175).

${ }^{14}$ Propos recueillis à Merzouga, novembre 2005. 
opposition à ceux ne recevant que l'eau des pluies. On retrouve cette distinction physique en Kabylie, où le roseau marque la limite entre terres cultivées et terres incultes (Yacine 1993: 134). Les haies de roseaux et de palmes croisées servent aussi, en milieu oasien, à diminuer l'évaporation, par l'ombre qu'elles procurent aux cultures (Champault I969: I2I).

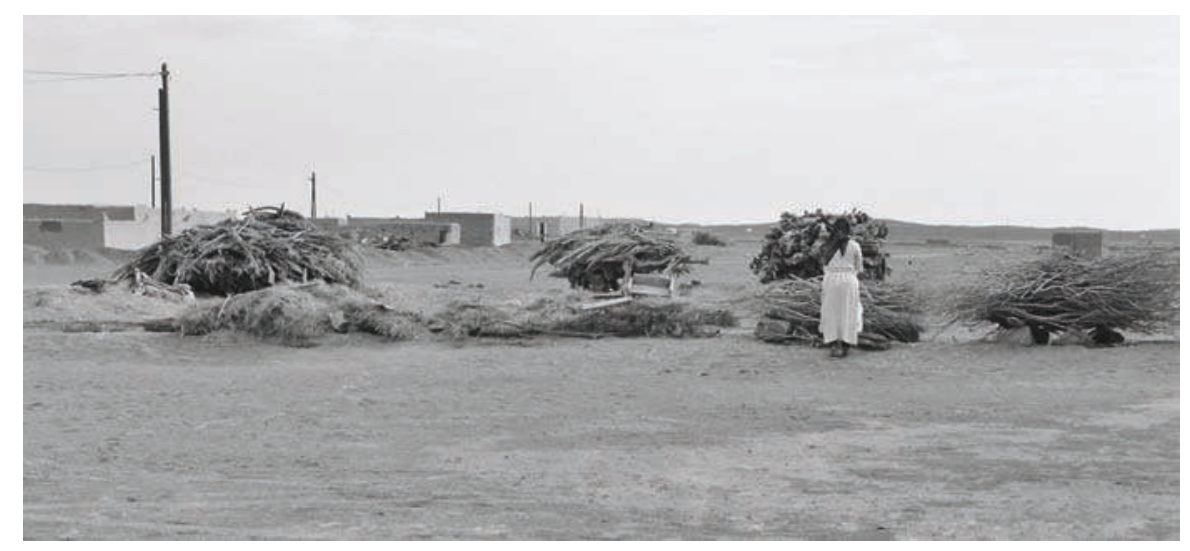

Figure 5. Bois destiné au four à pain. Merzouga (cliché M.-L. Gélard, 2005).

\section{Mesure de l'eau, mesure des corps}

Si le roseau permet de délimiter des espaces en les protégeant, il est aussi un instrument de mesure. Dans de nombreux pays du bassin méditerranéen, la canne de roseau est utilisée comme outil de mesure de l'eau, dans les systèmes d'irrigation par retenue (bassins réservoirs), c'est le cas au Portugal, en Espagne et au Maroc (Wateau 200I: I53-I6I). La tige de roseau est marquée par des «bâtonnets de roseau taillés et insérés et/ou des lacets

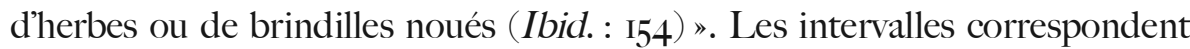
aux parts d'eau des différents ayants droit. "La canne visualise l'inégalité des richesses entre les bénéficiaires d'eau” (Ibid.: 153). Dans tout le Sud marocain, on relève diverses techniques de conservation de l'eau, barrage de retenue, conservation dans des bassins, dérivation d'eaux permanentes, etc.

«Dans les cas où l'eau est accumulée dans un bassin avant d'être livrée aux parcelles, la mesure de quantités d'eau intervient directement dans la mesure des parts. Ici, ce à quoi a 
droit chaque usager, c'est dans tous les cas une part du volume de l'eau accumulée (dont la variation est directement perçue par lintermédiaire de la variation de la hauteur d'eau dans le bassin) et non une quantité d'eau déterminée. Cette part est exprimée dans une unité constante ou variable " (Chiche $1984:$ 253-254).

Le roseau sert également à la mensuration du corps, celui du mort avant de creuser la tombe (Yacine 1993: 134) ainsi qu’à celui du nourrisson. On accorde une extrême importance aux modifications physiques du corps des nouveaux-nés. En effet, de la naissance jusqu'au quarantième jour, le nourrisson est en danger permanent du fait de la présence des jnûn à ses côtés, lesquels tentent de s'emparer de lui. C'est la croyance répandue en «l'enfant changé ", puisque les jnûn substituent leur propre progéniture aux nourrissons humains. Très souvent, ces enfants "changés" meurent avant d'atteindre leur quarantième jour. Aucune modification physique n'accompagne la subrogation, par contre, l'arrêt de croissance manifeste du nourrisson trahit l'échange. La vérification systématique de la croissance du nourrisson est faite à l'aide d'une tige de roseau, utilisée comme toise. En Kabylie, les mesures successives permettent de savoir si l'enfant a été victime du mauvais œil :

«On prend les mensurations (plusieurs fois) et on fait un nœud à chaque fois (sept fois si possible). Les fils sont ensuite comparés. Si tous les nœuds ne se retrouvent pas au même niveau on dit que l'enfant est diminué (yenqes) et done a été victime du mauvais oil (degs tit)»(Yacine $1993: 134)$.

Tassadit Yacine, signale également l'utilisation du roseau pour prendre les mensurations de la jeune mariée. La partie dépassant du corps est coupée. Avant la nuit de noces, ce roseau est fendu en deux et doit demeurer caché jusqu’à la défloraison, nous y reviendrons.

\section{L'enclos : tafergant}

Omniprésent dans la construction des maisons, la protection des terres cultivées, la mesure des droits d'eau et la mensuration des corps, on retrouve à nouveau le roseau dans l'élevage du bétail. Chez les Aït Khebbach, le mot " tafergant" (de la racine berbère $\mathrm{FRG}^{15}$, de afrag, la haie, la clôture) désigne à la fois une prohibition d'alliance entre des fractions et l'enclos ${ }^{16}$ situé à proximité de la tente (taghmt) ou de la maison et dans lequel les petits, non

${ }^{15}$ FRG ou FRQ exprime l'idée de fendre ou de séparer. En hassâniyya, les termes frîg ou vrîg, formés sur cette racine, désignent le campement.

${ }^{16}$ Également relevé par Ahmed Haddachi $(2000: 157)$. 
encore sevrés, sont enfermés en attendant le retour des troupeaux en pâture. Il s’agit d’une sorte de barrière protectrice destinée à protéger les femelles et leur progéniture. Construit en roseau et en palmes ${ }^{17}$, l'enclos se présente sous la forme d'un cercle à ciel ouvert. Les tiges de roseau sont légèrement rabattues vers lintérieur servant ainsi de paravent tout en procurant de l'ombre au cheptel. Les roseaux sont fichés dans le sol, sur une hauteur d'environ I mètre 20. J'ai illustré ailleurs l'homologie entre le terme qui désigne une prohibition matrimoniale dérivée d’ancien pacte de colactation et l'enclos qui protège les petits encore à la mamelle (Gélard : 2003). Ici encore, le roseau est associé à la protection.

Chez les touaregs Kel Ferwan du Niger, Dominique Casajus (1987: 82) décrit ce même enclos destiné au petit bétail, lequel est constitué de branchage et de deux compartiments circulaires permettant de séparer les petits des adultes. Le petit enclos est appelé egrur, l'ensemble, comme chez les Ait Khebbach, se nomme afarag. Il souligne la concordance entre le nombre d'enclos - il en existe un ou deux par tente (famille) - , et la cohésion du campement ${ }^{18}$. En effet, l'augmentation du nombre d'enclos témoigne du fait que le campement va bientôt se scinder pour former d'autres campements distincts. Les enclos et leur état permettent aussi de connaitre la durée d'un campement dans un lieu déterminé.

"Sur le site d’un campement, même abandonné depuis des années, quelques branches restées là signalent toujours l'emplacement d'un ancien enclos. C'est en considérant les enclos qu'on peut done voir vieillir le campement. Leur aspect révèle depuis combien de temps il est installé sur son site actuel, tandis que leur nombre permet de savoir à quel stade de son existence il en est " (Casajus I987: 65).

Le calame : l'écriture thérapeutique

Avant d'aborder les représentations qui découlent de l'omniprésence de l'utilisation matérielle du roseau dans la culture saharienne, il reste à évoquer un dernier emploi qui illustre le fait que les représentations ne sont pas de

17 Parfois en broussailles. Voir au Maroc, les descriptions des différents enclos proposés par Emile Laoust $(1935: 176)$.

18 Au sein du monde touareg, Edmond Bernus (1985) souligne que les iklan (captifs) sont parfois appelés deffar-afarag, soit «derrière la haie », en référence à l'installation de leur tente dans les campements, qui se situe au-delà des enclos à bétail. 
simples symboles mais investissent des rapports sociaux, devenant par là même agissantes ${ }^{19}$.

Le calame, en arabe qalame, la plume, a longtemps été constitué d'un morceau de roseau taillé ${ }^{20}$. On sait l'importance de l'écrit en islam, le calame est aussi le titre d'une sourate. Il symbolise l'émanation première de l'œuvre divine (Chebel 1995: 80). Le roseau, utilisé comme plume en raison notamment de ses vertus absorbantes (il retient l'encre dans ses fibres végétales), est l'instrument principal du faqih lors de la rédaction d'amulettes destinées soit à la protection, soit à la guérison. Très fréquentes dans tout le Maghreb, les amulettes du faqih sont coutumières en milieu rural marocain. Dans le Tafilalt, les faqih les plus réputés sont ceux de Zagora auxquels les Ait Khebbach ont fréquemment recours pour des nécessités thérapeutiques ${ }^{21}$. La lettre que trace le roseau ne doit pas être vue, les amulettes sont généralement insérées dans un petit morceau de cuir cousu. Le calame est l'instrument de l'écriture. L'expression commune de ces actes rituels est la suivante «il a écrit» ou "il faut que le faqih écrive quelque chose", l'efficacité thérapeutique dérive, certes de la puissance de l'acte d'écriture mais aussi et surtout de l'ingestion de la lettre (Djéribi 1993 : 9495). En effet, dans de nombreux cas, ce que l'on nomme «lincantation écrite " nécessite que l'ordonnance, établie par le maitre d'école coranique par exemple, soit ingérée par le patient. Ces formes d’ingestion, sur lesquelles nous reviendrons à propos du rituel de fermeture du corps de la jeune fille, renvoient explicitement à la prévalence du corps et du savoir "incorporé »22 (Bourdieu I980: III-I34), autrement dit jamais détaché du corps renvoyant à la transmission par contact. En ce sens, l'efficacité thérapeutique et/ou protectrice des amulettes fonctionne selon le même schéma de pensée puisqu'elles ne doivent pas être détachées du corps avant une certaine

\footnotetext{
19 Sur ce point particulier, voir les nombreux développements proposés par Maurice Godelier $(1978,2004)$. Il existe deux composantes des réalités sociales, la part matérielle (outil) et la part idéelle (représentation), or ces représentations sont nécessaires à la mise en œuvre, en action des moyens matériels.

20 Il demeure l'outil de la calligraphie. Le bec du calame est taillé au sommet du roseau (partie la plus dure).

${ }^{21}$ Certains lettrés du village confectionnent aussi les amulettes en inscrivant des sourates du Coran. Moins prestigieuses, elles sont souvent considérées comme de moindre efficacité et font plutôt office de gage de protection (nourrisson), d'autres permettent de se débarrasser des termites qui rongent les poutres des habitations.

22 «Ce qui est appris par corps n'est pas quelque chose que l'on a, comme un savoir que l'on peut tenir devant soi, mais quelque chose que l'on est. Cela se voit particulièrement dans les sociétés sans écriture où le savoir hérité ne peut survivre qu'à l'état incorporé » (Bourdieu 1980 : 123).
} 
période (c'est le cas des nourrissons jusqu’à leur quarantième jour). Et lorsque l'amulette est retirée, très souvent elle est censée se détacher seule du corps, on prend soin de ne jamais laisser passer quiconque entre celle-ci et la personne qu'elle protégeait.

\section{Des nécessités d'ordre technique aux représentations symboliques}

Le roseau occupe différentes fonctions techniques lesquelles s'associent explicitement à la protection. La terrasse en roseau préserve lintimité de l'habitation, mais elle permet surtout de protéger des aléas climatiques (isolation thermique). Le roseau protège la terre cultivée de l'ensablement, conçu et perçu comme la principale menace en milieu oasien saharien. La toise de roseau est également signifiante face à l'existence des individus, puisque la mesure du corps du nourrisson permet de constater visuellement les signes de sa croissance, considérés comme les preuves manifestes de son humanité et de sa santé.

Ce sont encore les vertus protectrices du roseau qui sont évoquées à propos de l'enclos qui sépare les petits des animaux adultes. En effet, cet enclos n'a pas les mêmes caractéristiques que celle habituellement dévolue aux pacages du bétail, c'est-à-dire l'empêcher de se disperser, car, dans le cas précis du tafergant, les roseaux préservent les petits encore à la mamelle.

Au niveau des représentations, le terme "tafergant» est usité comme la forme la plus courante d'un interdit matrimonial entre fractions anciennement unies par un pacte de colactation collectif. En d'autres termes, l'utilisation métaphorique d'un terme référé à l'enclos protégeant les petits non encore sevrés et marquant la nécessité d’une séparation stricte entre individus issus de groupes colactés manifeste ici encore le rôle " analogique " protecteur du roseau. En effet, les conséquences d'une alliance entre individus tafergant agit par une sorte de malédiction conditionnelle dont on impute l'effet aux pouvoirs du "lait", entité sommitale qui agit sur les contrevenants soit par la mort pure et simple (du couple et/ou des membres de la famille proche) soit par une atteinte sur leur progéniture ou sur le patrimoine (terres, bétail, etc. $)^{23}$.

23 Si aujourd'hui les plus jeunes rapportent avec un certain amusement ces «croyances » des anciens, on constate dans les faits que peu d'individus tafergant se marient. Deux exemples les plus significatifs au sein du 


\section{Roseau et rituels : l'inaccessibilité au corps de l'épouse}

Commun à de nombreuses régions du Maghreb, le rituel appelé tkhaft consiste à rendre impossible la relation sexuelle. Ce rituel dit «de fermeture ", le terme est dérivé de la racine arabe KF, arrêt, stop, est censé obturer le sexe féminin. Les femmes utilisent ces procédures rituelles pour leurs filles célibataires et, ce, souvent dès leur puberté.

Parmi les procédés de fermeture les plus courants on observe le fait, pour la jeune fille de passer trois fois sur un moulin en pierre les yeux fermés, ou une fois sous un métier à tisser ${ }^{24}$. Pour inverser l'action magique, à la veille de son mariage, la jeune fille doit se laver sur l'ensemble des outils du tissage (peigne, rouet, etc.).

"La technique connue sous le terme śfah', qui consiste à bloquer rituellement l'accessibilité du vagin, augmente ce "muselage moral" : l'homme étant censé échouer dans ses entreprises, arrêté par cette barrière contre laquelle son pénis "ricoche" " (Chebel I984: 59).

La partie mobile (baguette) qui sert à distinguer les fils pairs et impairs du métier à tisser ${ }^{25}$ est très généralement constituée d'une tige de roseau. Comme le souligne Tassadit Yacine (1993: 134), les fonctions symboliques du roseau sont multiples, et s'il représente la virginité de la jeune fille :

« Il craque, il se fend, il est beau mais fragile. Il est surtout le symbole de l'âme (rruh: il monte et descend dans le métier, comme le souffle de la vie dans le corps de l'homme). "

À Merzouga, on relève un autre procédé rituel qui consiste à faire boire à la jeune fille de l'eau mélangée à la petite membrane blanche que l'on trouve à lintérieur du roseau (aghanim en berbère). La membrane sépare les différents segments de la canne. Les tiges de roseau présentent en effet une

village de Merzouga servent toujours de modèle aux éventuels candidats. L'un rapporte la paralysie immédiate du jeune marié lors de l'arrivée de l'épouse sous la tente nuptiale (la présence de la tente y compris en milieu sédentaire est une règle à laquelle aucune cérémonie de mariage ne déroge à Merzouga mais aussi dans les villages alentour), le second rappelle la succession des décès enregistrés parmi les membres de la famille du marié : père, mère puis sœur en l'espace d'à peine une année.

24 À ce propos voir le rite de tasfiha décrit par G. Libaud (1986:137-140) en Tunisie, où c'est le métier à tisser qui sert de frontière entre l'extérieur de l'habitation et l'intérieur où demeure protégée la jeune fille non mariée. L'inversion du rite de fermeture est matérialisée à l'occasion du mariage par le fait de coucher le métier à tisser sur le sol.

25 Il s'agit du métier à tisser «nord-africain » selon la terminologie d'André Leroi-Gourhan (1971 : 288) : «Audessus des lisses, une seule baguette assez forte, prend les fils impairs. Il suffit d'élever la baguette pour ouvrir le pas (pairs dessous), de l'abaisser en la repoussant pour ouvrir le pas inverse (pairs dessus); la trame est passée à la main ». 
structure spéciale, où les segments sont creux entre les entre-nœuds et plein aux nœuds ${ }^{26}$. La membrane se situe à chacun des nœuds. Ce procédé semble apparenté à certaines techniques contraceptives, sorte de stérilité voulue pour une durée précise. Les femmes recueillent des grains d'orge tombés de la bouche d'une mule, animal stérile par nature, ou prélèvent de la corne de leur sabot (Legey 1926: 75).

Lingestion de la membrane du roseau est un des procédés rituels communs à l'ensemble des tribus Aït Atta sahariennes du Tafilalt. On retrouve différentes méthodes dingestion dans les prescriptions rituelles magiques telles que décrites par Edmond Doutté (1908). Muriel Djéribi (1993: 94) en relate les occurrences caractéristiques mettant en parallèle lincantation, l'écriture et au final l'ingestion de la lettre.

"À l'évidence, incanter, oralement ou par écrit, c'est "écrire sur...", c'est-à-dire, créer une surface dinscription. Ensuite, que cette inscription s'accompagne d'un traitement très particulier de la lettre, qui consiste à l'incorporer par le moyen d'un liquide ou de toute nourriture qui aurait été en contact avec elle. »

On pourrait ainsi supposer que la membrane qui sépare les sections du roseau serait la représentation métaphorique de l'hymen, son ingestion permettrait de créer cette fermeture du corps de la femme en préservant intact l'hymen. La ressemblance entre l'hymen, membrane qui obstrue partiellement l'orifice vaginal, et la sorte d'opercule du roseau est conséquente. Le procédé rituel fonctionnerait ainsi par analogie.

Si le roseau implique la protection de la virginité, il sert aussi à prémunir les mariées du mauvais œil (Yacine 1993: 134). Il est ainsi pleinement associé à la fertilité comme en témoigne également son utilisation dans les rituels agraires pour favoriser le retour de la pluie.

\section{Rites de rogations pour la pluie}

Dans la confection du mannequin (tlaghnja) usité dans les rites d'obtention de la pluie, les bras sont constitués de cuillères en bois attachées sur une armature en roseau figurant le corps. Les jeunes filles le promènent en cortège dans les rues du village. Luutilisation du roseau est systématique à l'occasion de ce rituel particulier et ce dans l'ensemble du Maghreb (Doutté I905 : 383; Laoust $1920: 205$ ). L'association roseau/eau/pluie est récurrente. Par ailleurs, ce que d'aucuns ont improprement qualifié de "fiancée " de la

${ }^{26}$ Pour plus de détails, voir la description botanique faite par Paul Ozenda (1983 : 143-144). 
pluie - parfois nommée "tlaghnja»-, est en réalité la figuration d'une femme mariée ${ }^{27}$, et féconde. L'importance de la fécondité et des valeurs natalistes qui lui sont associées nécessitant une représentation féminine du mannequin dont la fécondité (il s'agit d’une épouse/mère) est avérée, ce qui n'est naturellement pas le cas d'une fiancée.

Toujours associé à la fertilité, le roseau est utilisé à l'occasion des rituels de la naissance. Bien que la coupe du cordon soit aujourd'hui souvent faite à l'aide d'un couteau, il est encore d'usage de sectionner ce dernier à l'aide d'un fragment de tige de roseau fendu en deux, qui donne une lame acérée d'une efficacité extrême. Les femmes l'utilisent car il est perçu comme un gage de fécondité future pour le nouveau-né.

On connaît les vertus coupantes de l'arundo donax, ce que décrit Claudine Fabre-Vassas (1983 : 13) évoquant à propos de la chirurgie pastorale (perforation de la panse des brebis) et de la castration des porcs en Provence, lutilisation de ces couteaux végétaux.

Dans le Sud marocain, lors de la préparation de la découpe de la viande du sacrifice, le roseau est utilisé comme outil perforateur de la peau, au niveau de l'un des membres antérieurs de l'animal. Le procédé permettant ensuite en soufflant de l'air par l'orifice ainsi crée, une séparation plus facile de la peau de l'animal. En frappant alors sur les flanes gonflés de la carcasse, la peau se détache de la chair ${ }^{28}$.

\section{La mariée du roseau (tislit n-aghanim)}

Offrant un parallèle avec le mannequin des rites de rogations pour la pluie, la confection des poupées des fillettes est le plus souvent réalisée sur l'armature d'un roseau.

En milieu berbérophone, les fillettes confectionnent des poupées appelées "tislit» (la mariée) en utilisant deux morceaux de roseau croisés, l'un pour figurer le corps et le second les bras. Elles habillent l'armature en reproduisant le costume traditionnel des mariées et miment ensuite les cérémonies du mariage. Ce jeu, très répandu et toujours d’usage, occupe les

\footnotetext{
27 Pour plus de détail sur cette distinction «femme/fiancée », voir M.-L Gélard (2006).

28 Il convient de dépecer avec le plus grand soin les animaux, à plus forte raison celui du sacrifice de l'Aïd el Kebir. À Merzouga, la peau est conservée et tannée par les femmes, contrairement à la pratique en milieu urbain où les peaux sont vendues, celle du mouton du sacrifice doit rester propriété de la famille, elle ne peut entrer dans le réseau économique. Les plus anciennes y sont très attentives.
} 
fillettes ${ }^{29}$ dès leur plus jeune âge. Elles récupèrent des morceaux de tissus auprès des femmes de leur entourage. Il arrive parfois que le marié soit aussi représenté par l'une de ces figurines mais dans la majeure partie des cas qui mont été donné d’observer (Tafilalt, Haut Atlas et région de Rabat) la mariée est la figure la plus fréquente. On substitue parfois au roseau, un morceau d'os. Compte tenu des techniques même de fabrication, la poupée est nommée " tislit n-aghanim " (la mariée du roseau) ou « tislit n-akhqjig " (la mariée de l'os).

Cette association entre os et roseau offrant l'un à l'autre un objet de substitution est intéressante. Cette concordance est aussi relevée par Claudine Fabre-Vassas (1983: I5), dans un tout autre contexte (en pays de Sault), dans les Pyrénées. Elle souligne notamment la relation métaphorique du roseau qui remplace $\operatorname{los}^{30}$.

En milieu rural marocain, le roseau est utilisé pour immobiliser les membres fracturés, sa nature à la fois souple et résistante permet, dit-on, d'éviter de bloquer la circulation du sang. La mise en relation du roseau avec le système humoral (sang et lait), en tant que régulateur, est soulignée par Claudine Fabre-Vassas. Dans cette perspective, l'auteur évoque également les différentes vertus médicinales qui lui sont imputées depuis les Grecs, à savoir ses pouvoirs cicatrisants et le fait quill puisse faire «passer le lait ». De la sorte, en décoctions (feuilles, tiges et racines de la plante) il permettrait de tarir le lait des nourrices.

"La recommandation est générale, le roseau est un «antilaiteux ", sa racine surtout, pleine de nodosités, bride l'une des manifestations les plus visibles de la fécondité ; cette croyance, toujours très vivace, vient ici confirmer ses effets stérilisants » (Ibid. : I7).

29 Le tourisme étant fort développé en milieu rural marocain, les jeunes filles des villages confectionnent souvent des poupées de ce type qui sont ensuite proposées aux touristes moyennant une dizaine de dirhams (équivalents à un euro). On observe ensuite l'intervention des hommes dans cette vente, c'est le cas des nomades Aït Khebbach dans le Sud. Dans la mesure où, à l'origine, ces poupées sont exclusivement confectionnées et utilisées de manière ludique par les fillettes, on assiste alors à une sorte d'inversion des genres, impensable dans le cadre traditionnel de l'entre-soi, où c'est un homme adulte qui prend en charge cette vente. L'opposition entre la vie au village ou sous la tente et celle qui impose le contact temporaire avec des Occidentaux demeure très forte, l'hermétisme entre ces deux modes de vie permet de nombreuses inversions dans les fonctions et les attributions traditionnellement dévolues aux femmes, sans que ceci soit l'objet de commentaires ou de moqueries.

30 En Kabylie, la flûte peut être constituée d'un morceau de roseau mais aussi d'un os. C'est le cas dans le célèbre conte «la flûte d'os" (Amrouche 1982 : 79-82) où un crime fratricide longtemps demeuré secret, est révélé par une voix miraculeuse venue d'une flûte d'os. 
Il ne s'agit pas ici de dresser un bilan comparatiste des vertus attribuées au roseau, mais il parait important de souligner l'opposition de ces principales fonctions telles qu'elles sont évoquées par la médecine des "simples" et les qualités attribuées au roseau en milieu saharien. Si la systématisation des fondements de la pensée hippocratique par la philosophie et la médecine grecques édifie «la pensée thérapeutique la plus durable de l'histoire occidentale" (Lieutaghi 1986:47), on peut supposer que d'autres modes d'appréhension se développent ailleurs ${ }^{31}$. Ainsi, si le roseau est susceptible de «faire passer le lait» en Europe, au Maghreb, au contraire il est pleinement associé d’une part à la fécondité (coupe du cordon ombilical, rites de rogation pour la pluie) et d'autre part à la lactation et à aux formes d'allaitement «surnaturelles ». Loin de couper le lait, il aurait la vertu de le protéger, comme c'est le cas pour l'enclos destiné aux petits à la mamelle. Métaphoriquement, il empêche les conséquences néfastes d'une union d'individus apparentés par le lait (tafergant). Voyons son rôle dans une des formes d'allaitement extraordinaires, celle de la lactation post-mortem, mise en scène dans la littérature orale.

\section{La vache des orphelins}

Au Maghreb, le conte de La Vache des orphelins est très répandu, on en connait des versions multiples en Algérie, au Maroc et en Tunisie. Nous n'aborderons pas dans le contexte de cette contribution les différents motifs narratifs, l'histoire rapporte les faits suivants. Dans une famille de paysans pauvres, l'épouse encore fort jeune décède et laisse ses deux enfants à la charge de leur père. Juste avant de s'éteindre, elle fait promettre ${ }^{32}$ à son mari de ne jamais vendre l'unique vache quils possèdent ${ }^{33}$. L'homme se remarie très vite, avec une femme ayant déjà une fille d'un premier mariage (dans d'autres versions, la seconde femme met au monde une fille). Cette seconde femme, dont les versions s'accordent unanimement sur l'extrême méchanceté, souhaite se débarrasser des enfants du premier lit de son mari lesquels

\footnotetext{
31 Une recherche en cours propose la comparaison entre la systématisation de la théorie hippocratique des humeurs et les modalités d'appréhension de celles-ci dans des sociétés supposées ignorer cette problématisation. De la sorte, la reprise par Galien de la pensée hippocratique comme assise de la médecine médiévale (Lieutaghi 1986 : 49) nous parait ouvrir des perspectives de recherches comparatives intéressantes.

32 Il est parfois rapporté qu'elle émet un «interdit de vente».

33 Dans une autre version, la mère lègue à ses enfants la vache.
} 
doivent leur subsistance à la production laitière de la vache. La marâtre finit par obtenir du père la vente de la vache ${ }^{34}$, mais à son grand étonnement, elle constate que les enfants non seulement ne dépérissent pas, mais continuent de grandir. Contrariée, elle enjoint sa fille de suivre ses demi-frères pour élucider ce mystère. Celle-ci s'exécute et suit les enfants partis pleurer sur la tombe de leur mère. Dès leur arrivée, deux morceaux de roseau surgissent du tombeau, l'un offrant du lait et l'autre du miel. Les enfants se rassasient alors des deux substances nourricières ${ }^{35}$. La fille attend leur départ et s'approche de la tombe pour goûter aux mets précieux, mais à la place du lait et du miel, coulent en abondance du sang et du pus ${ }^{36}$, parfois même du fiel ou du goudron perçu comme l'opposé du lait, pour sa noirceur. On sait limportance du motif du lait noir dans la littérature orale notamment comme « expression d’une maternité malfaisante» (Lacoste-Dujardin I982 : 262).

On voit comment, dans ce conte célèbre, le roseau protecteur est pleinement illustré dans son rôle nourricier et salvateur. On le retrouve également dans les sacrifices destinés à conjurer le mal.

\section{Sacrifices conjuratoires et territorialisation du mal}

Derrière la plus grande dune du village d'Hassilbyed, existe un lieu que les femmes considèrent comme sacré. Il s’agit d'une petite roselière, plantée dans le sable «depuis toujours" dit-on (fig. 6). C’est en général le jeudi que les femmes choisissent de sy rendre.

\footnotetext{
34 La marâtre prétextant que celle-ci avait donné un coup de sabot à sa fille. La vente n'est pas systématique, dans certains récits, on rapporte que personne au marché ne voulut déposséder les orphelins et que la marâtre exigea de son époux qu'il égorge la vache.

35 «Les orphelins se rendirent alors au cimetière pour pleurer sur la tombe de leur mère. Mais voici que deux roseaux s'élevèrent de la tombe. L'un donnait du beurre et l'autre du miel. Les enfants se penchèrent et se mirent à les sucer alternativement. Grâce aux roseaux, les orphelins qui avaient dépéri redevinrent blancs et roses» (Amrouche $1982: 57$ ).

36 Également souligné par Camille Lacoste-Dujardin (1982 : 254) ce conte populaire très répandu apparaît sous des titres divers: Les Deux Orphelins jumeaux, Les Enfants de la marâtre, La Marâtre et les orphelins, La Femme et les deux enfants. L'auteur (2000: 73) relève aussi l'utilisation du roseau comme procédé d'allaitement post-mortem : «Il pourrait même arriver, vient-on à imaginer dans la culture kabyle, que le lait maternel puisse être transmis par-delà la mort. C'est ce qui est narré dans un conte très populaire qui montre deux orphelins nourris sur la tombe de leur mère par deux roseaux qui en surgissent, leur dispensant lait et miel ».
} 


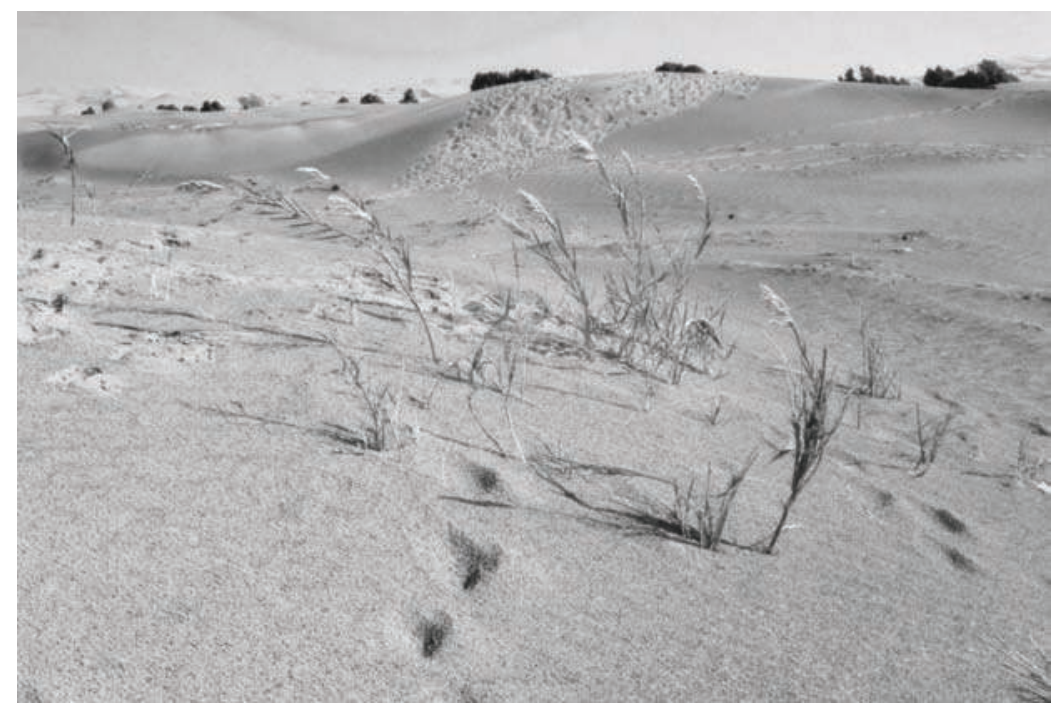

Figure 6. Roseaux sauvages dans les dunes de sable dont le développement crée parfois de véritables roselières (cl. M.-L. Gélard, 2005).

Comme à Merzouga, la dune la plus haute est personnalisée, on la nomme Lalla Ilalia et les femmes lui attribuent des pouvoirs magiques divers ${ }^{37}$. Ces pratiques sont à rapprocher du culte des saints et de linfluence des croyances berbères préislamiques (génies des lieux notamment). On sait l’importance des transformations d'anciennes légendes réinvesties par de nouvelles significations (islam populaire). Les femmes effectuent une première visite sur le lieu, en demandant guérisons et bienfaits divers. Elles accrochent alors un morceau de tissu de leur vêtement sur l'une des cannes de roseau et décident également d'une période d'attente (une semaine ou un mois) au terme de laquelle leur souhait doit être exaucé. Si c'est le cas, elles reviennent et au milieu des roseaux égorgent un $\operatorname{coq}^{38}$, le sang devant se mêler au végétal

37 Elle protège la communauté villageoise et ce sont les femmes qui s'y rendent pour demander ses bienfaits. En contrebas de cette dune pousse un tamaris de forme originale (sorte de pont dessiné par le tronc), au pied duquel sont effectués différents rituels.

38 Les femmes n'effectuent pas le sacrifice, la tâche incombe alors au plus âgé des garçons présents avec elles. À partir d'une dizaine d'années, le garçon est en mesure d'effectuer le sacrifice des poulets. À propos de la fréquence des sacrifices de gallinacés en Afrique du Nord, voir Emile Dermenghem (1954 : 157-161). Au Sahara 
et maculer quelques tiges de roseau. La consommation de la viande ne s'effectue pas sur place et les femmes ayant participé au sacrifice ne doivent pas en manger. Ce rituel sacrificiel a pour fonction de permettre une expulsion du mal. En effet, les femmes expliquent que le sacrifice et le sang versé sur ce lieu particulier permettent d'y inserire le mal, via le sang comme marqueur physique, afin de l'empêcher de revenir chez soi. Ces rites d'expulsion s'apparentent, tout au moins partiellement, aux définitions de la fonction d'émissaire telle que décrite par René Girard (1998 : 122) mais ici précisément par linstrumentalisation sacrificielle. En effet, le coq n’est pas la "victime émissaire", il est lïnstrument de la translation, soit l'équation: fixation du mal sur la victime sacrificielle, dépôt/expulsion de ce dernier, et maintien, donc éloignement des différents maux dans un lieu géographique circonscrit, ici une petite roselière. L'éloignement physique apparaissant comme le corollaire de la simple séparation ${ }^{39}$. Le fait que le lieu soit précisément constitué de roseaux, nous paraît ici signifiant, car comme nous l’avons précédemment souligné, les roseaux sont explicitement associés à l'enclos (la clôture, la séparation). Les roseaux ne sont pas ici sacralisés mais servent la relation entre végétal et sacré comme l'a montré Emile Dermenghem (1954 : 136-137) à propos des arbres et des bois.

En milieu berbérophone, on relève une multiplicité de rituels d'expulsion du mal qui ne nécessitent pas forcément de sacrifices sanglants. C'est le cas, chez les Idaw Martini (Anti-Atlas central) lors de l'utilisation d'un végétal anthropomorphe, considéré comme "humide ", tige de maïs immature ou roseau sur lequel on transfert symboliquement son mal en y attachant un morceau d'étoffe (El Alaoui 2002 : 309). La fonction du végétal est ici encore de protéger dans la mesure où il « retient " les maux, établissant une nouvelle disjonction entre mal/individu et/communauté.

Les usages du roseau dans le Tafilalt sont d'une grande variété, à la fois dans leur utilisation technique mais aussi comme symbole et comme vecteur de fonctions rituelles explicitement dérivées de la protection. Le roseau protège certes de la chaleur et de l'ensablement, mais il a surtout un rôle éminemment salvateur, c'est le cas dès la naissance puisqu'il est l'instrument

occidental, le sacrifice du coq est valorisé et l'on pense que le jour de la mort, il transportera l'âme du croyant (Agabi 1996 : 2590).

39 En Kabylie, le rite d'expulsion se nomme asfel, le terme désignant à la fois le rite proprement dit et l'objet précis qui sert de support au transfert et à l'expulsion du mal (Abrous 1989 : 961). 
de la mesure des modifications du corps du nourrisson menacé de subrogation létale. Il intervient comme le protecteur des denrées nourricières du groupe (cultures et reproduction du cheptel animal), permettant leur fertilisation via les rites de rogations pour la pluie où il est un élément central. Et enfin, il permet de circonscrire divers maux, les éloignant de la communauté par cette sorte de pouvoir d'attraction qu’il manifeste autour de la fonction même de séparation quili initie.

L'utilisation technique et symbolique du roseau s'applique dans des lieux à sources d'eau permanentes, il serait ainsi intéressant de voir, dans la perspective d'un comparatisme raisonné, ce qu'il en est dans d'autres régions, celles à nappes phréatiques profondes où le roseau ne pousse pas ou très peu (Mzab, El Goléa, Bas Saoura). Dans ces cas-là, c'est le palmier qui est utilisé aussi bien pour la construction des terrasses que pour faire des clôtures. Les représentations sont-elles alors identiques ou les caractéristiques physiques du palmier illustrent-elles d'autres significations symboliques? Ainsi, lillustration du palmier "anthropomorphe " tel que le décrit Geneviève Bédoucha (I987: I07-II3) à propos des oasis tunisiennes évoquant la profonde intimité de l'homme et de l'arbre.

L'homme lui donne la parole, lui prête ses propos, ses sentiments, engage parfois un dialogue, le prend à témoin. Et le moment où il grimpe à l'arbre pour la fécondation est sans doute le plus chargé dintimité et le plus imprégné de symbolisme. Le palmier est femme et l'homme vient le trouver, porteur du pollen de l'arbre mâle (ed-dokkâr) et combler son attente (Ibid. : II2).

Sil convient de distinguer le roseau, en tant que végétal et les discours et représentations qu'il suscite, le lien ou mieux l'effet de miroir souligné entre son utilisation technique et les rapports sociaux auxquels il imprime valeur de protection nous paraissent ouvrir des perspectives intéressantes à l'étude des relations entre les individus et leur environnement naturel. Le territoire de la tribu des Aït Khebbach, caractérisé par un climat saharien et par une topographie où alternent formation dunaire et plateau caillouteux, témoigne d'un environnement hostile, où l'élevage (pâturage) et l'agriculture (irriguée) ne sont rendus possibles que dans des lieux restreints, lieux mêmes où l'on relève la présence du roseau. De la sorte, c'est la détermination écologique qui nous paraît témoigner de linntérêt porté à celui-ci, sa présence illustrant les possibilités d'existence même du groupe. Le roseau protège 
physiquement et métaphoriquement la société, de l'espace non habité, du vide... Et comme le souligne Augustin Berque (2000) sil manque à l’ontologie une géographie et à la géographie une ontologie: «Être c'est forcément être quelque part ».

M.-L. G.

\section{Références}

Abrous, Dahbia

1989 "Asfel. Sacrifice rituel (Kabylie) ", Encyclopédie Berbère VII : 96I962.

Agabi, C.

1996 "Égorgement ", Encyclopédie Berbère XVII : 2589-259I.

Amrouche, Taos

1982 Le Grain magique. Contes, poèmes, proverbes berbères de Kabylie. Paris : Maspéro.

Benfoughal, Tatiana

1996 «Du palmier dattier à la matière plastique. Tradition et mode dans la fabrication des vanneries sahariennes ", Cahiers de l'IREMAM 7-8:57-78.

Bernus, Edmond

1985 "Afarag (pl. Ifergan) (touareg) ", Encyclopédie Berbère II : 206-207.

Baroin, Catherine \& Pierre-François Pret

1993 "Le palmier du Borkou, végétal social total ", Journal des africanistes $63: 5-20$.

Bédoucha, Geneviève

I987 "L'eau, l'amie du puissant». Une communauté oasienne du Sudtunisien. Paris : Editions des Archives contemporaines.

Berque, Augustin

2000 Écoumène. Introduction à l'étude des milieux humains. Paris : Belin.

Boulay, Sébastien

2003 « Organisation des activités techniques féminines de fabrication de la tente dans la société maure (Mauritanie) ", Journal des africanistes $73^{(2)}:$ IO7-I2O. 
Bourdieu, Pierre

1980 Le Sens pratique. Paris : Éditions de Minuit.

Casajus, Dominique

I987 La Tente dans la solitude : la société et les morts chez les Touaregs Kel Ferwan. Paris : Éditions de la MSH.

Chebel, Malek

$1984 \quad$ Le Corps dans la tradition au Maghreb. Paris : PUF.

Chiche, J.

I984 "Description de l'hydraulique traditionnelle", in Nejib Bouderbala,

J. Chiche, A. Herzenni, Paul Pascon, La Question hydraulique.

Rabat : rapport de recherche non publié : 120-319.

Dermenghem, Émile

I954 Le Culte des saints dans lisslam maghrébin. Paris : Gallimard.

Djéribi, Muriel

1993 "Lincantation mythique : noms et écriture », Ethnologie française XXIII : 94-IO3.

Doutté, Edmond

I908 Magie et religion dans l'Afrique du Nord. Paris : Maisonneuve.

El Alaoui, Narjys

2002 "L'action rituelle pour la pluie. Anti-Atlas-Maroc », in E. Katz, A. Lammel et M. Goloubinoff (eds), Entre ciel et terre. Climat et sociétés. Paris : IRD-Ibis Press : 299-319.

Fabre-Vassas, Claudine

I983 "Le charme de la syrinx " L'Homme XXIII : 5-39.

Fennane, M. Mohammed (ed.)

1987 La Grande encyclopédie du Maroc, 5 tomes. Rabat : GEI.

Gaudry, Mathéa

1929 La Femme chaouia de l'Aurès. Étude de sociologie berbère. Paris : Geuthner.

Gélard, Marie-Luce

2003a Le Pilier de la tente. Rituels et représentations de l'honneur chez les Ait Khebbach (Tafilalt). Paris : Editions de la MSH.

$2003 \mathrm{~b}$ «Les représentations de la parenté. Prohibitions matrimoniales et substance lactée chez les Ait Khebbach (Maroc) ", Annales de la Fondation Fyssen 18 : I29-137.

2006 "La cuiller à pot (tlaghnja). Les rogations pour la pluie en Afrique du Nord ", Journal des Africanistes 76(I): Sahara, identités et mutations sociales en objets : 8I-IO2.

Girard, René

1998 La Violence et le Sacré. Paris : Hachette (I ère édition 1972). 
Godelier, Maurice

1978 «La part idéelle du réel. Essai sur lỉdéologie », L'Homme XVIII : I55I88.

2004 Métamorphoses de la parenté. Paris : Fayard.

Haddachi, Ahmed

2000 Dictionnaire de tamazight. Parler des Ayt Merghad (Ayt Yafalman).

Salé : Éditions Beni Snassen.

Hart, David Montgomery

198I Dadda 'Atta and his forty grandsons : the social-political organisation of the Ait 'Atta of southern Morocco. Wisbech : Middle East and North African Studies Press.

Hensens, Jean

I969 "Habitat rural traditionnel des oasis pré-sahariennes ", Bulletin économique et social du Maroc XXX(II4) : 83-IO7.

Lacoste-Dujardin, Camille

I982 Le Conte kabyle. Étude ethnologique. Paris : Maspéro.

2000 "La filiation par le lait au Maghreb ", L'Autre. Cliniques, cultures et sociétés I : $69-76$.

Laoust, Émile

I935 L'Habitat chez les transhumants du Maroc central. Paris : Éditions Larose.

Legey, Doctoresse

I926 Essai de folklore marocain. Paris : Éditions Geuthner.

Leroi-Gourhan, André

I97I L'Homme et la matière, Paris : Albin Michel (I ${ }^{\text {ère }}$ édition I943).

Libaud, Geneviève

1986 Symbolique de l'espace et habitat chez les Beni-Aissa. Paris : Éditions du CNRS.

Lieutaghi, Pierre

I986 «Le livre des simples médecines. Un printemps de la pensée médiévale du corps et de la nature ", Bulletin d’ethnomédecine 37 : 790.

Ozenda, Paul

$1983 \quad$ Flore du Sahara. Paris : Éditions du CNRS.

Wateau, Fabienne

200I «Objets et ordre social. D’une canne de roseau à mesurer l'eau aux principes de fonctionnement d'une communauté portugaise », Terrain 37 : I53-I6I.

Yacine, Tassadit

1993 Les Voleurs de feu. Éléments d'une anthropologie sociale et culturelle de l'Algérie. Paris : Éditions La Découverte/Awal. 